\title{
Single-shot high-resolution dual-broadband CARS interferometric lineshape spectroscopy
}

\author{
K. A. Vereschagin, ${ }^{1}$ V. V. Smirnov, ${ }^{1 *}$ O. M. Stel'makh, ${ }^{1}$ V. I. Fabelinsky, ${ }^{1}$ W. Clauss, ${ }^{2}$ \\ D. N. Klimenko, ${ }^{2}$ M. Oschwald ${ }^{2}$ and A. K. Vereschagin ${ }^{2}$ \\ ${ }^{1}$ General Physics Institute (IOFAN), Vavilov Str. 38, 119991 Moscow, Russia \\ ${ }^{2}$ German Aerospace Center (DLR), Space Propulsion Institute, Langer Grund, 74239 Hardthausen, Germany
}

Received 4 July 2004; Accepted 22 August 2004

\begin{abstract}
A high-resolution spectroscopic method that allows single-shot spectra of Raman-active molecular transitions in non-stationary media to be obtained with a resolution of $\sim 0.1 \mathrm{~cm}^{-1}$ was developed. The method is based on application of a Fabry-Pérot interferometer to spectral analysis of the CARS signal from Raman transitions with further detection of an interference pattern by a two-dimensional photo-matrix. The implementation of a dual-broadband (DBB) CARS configuration for effective spectral averaging over pump and Stokes laser spectra together with angular averaging of CARS intensities along the complete circumference of Fabry-Pérot interference patterns leads to a significant improvement of interferogram quality, so that nearly all of the single-shot interference patterns obtained for Q-branch lines of the hydrogen molecule in a gas probe volume became suitable for linewidth measurements. Copyright (c) 2005 John Wiley \& Sons, Ltd.
\end{abstract}

KEYWORDS: dual-broadband CARS; single-shot lineshape spectroscopy; linewidth measurement

\section{INTRODUCTION}

In applications of CARS spectroscopy devoted to thermometry in high-pressure, high-temperature $\mathrm{H}_{2} / \mathrm{O}_{2}$ combustion based on $\mathrm{H}_{2}$ Q-branch CARS spectra, the knowledge of $\mathrm{H}_{2}-\mathrm{H}_{2} \mathrm{O}$ collisional broadening coefficients together with their temperature and $J$ dependence is necessary for correct evaluation of gas temperature. ${ }^{1-4}$

Hydrogen line broadening coefficients at temperatures not exceeding $1800 \mathrm{~K}$ have been measured by Berger and co-workers ${ }^{5-7}$ for collisions with $\mathrm{N}_{2}, \mathrm{H}_{2}$ and $\mathrm{H}_{2} \mathrm{O}$. In order to perform such an investigation, they applied high-resolution scanning stimulated Raman gain techniques with continuous-wave (CW) single-frequency tunable pump sources to a stationary medium in a heatable high-pressure vessel. ${ }^{5,6}$ As they noted, the main factor that poses a limit to increasing the vessel temperature is the high chemical activity of water vapor at these high temperatures and static conditions. Possible alternatives to static vessel measurements are hydrogen Raman linewidth measurements directly in a hydrogen-oxygen burner where continuous refreshment of the medium takes place.

*Correspondence to: V. V. Smirnov, General Physics Institute (IOFAN), Vavilov Str. 38, 119991 Moscow, Russia.

E-mail:vvs@kapella.gpi.ru
In a previous paper, ${ }^{4}$ we reported on measurements of high-temperature collisional broadening coefficients of various $\mathrm{H}_{2}$ Q-branch lines broadened by water molecules that have been performed in a pulsed high-pressure $\mathrm{H}_{2}-\mathrm{O}_{2}$ burner. The values of $\mathrm{H}_{2} \mathrm{Q}_{1}, \mathrm{Q}_{3}, \mathrm{Q}_{5}$ and $\mathrm{Q}_{7}$ collisional broadening coefficients measured in our experiments ${ }^{4}$ at $2700 \mathrm{~K}$ coincide within the experimental error with the values extrapolated from the values measured at $1800 \mathrm{~K}$ in a heated cell filled with $\mathrm{H}_{2}$ and water vapor. ${ }^{5-7}$

Whereas in the case of single-shot CARS thermometry of $\mathrm{H}_{2} / \mathrm{O}_{2}$ flames, in the temperature range $300-2700 \mathrm{~K}$, experimental and extrapolated data measured ${ }^{4-6}$ together with model calculations ${ }^{7}$ can successfully be used, for hydrocarbon-fuelled flames the problem of correct temperature evaluation still persists. This is mainly due to the lack of knowledge of $\mathrm{H}_{2}-\mathrm{N}_{2}, \mathrm{H}_{2}-\mathrm{CO}_{2}$, etc. collisional line broadening coefficients at elevated temperatures and additional experimental complications arising from the necessity for simultaneous measurements of $\mathrm{H}_{2} \mathrm{O}, \mathrm{CO}_{2}$ and $\mathrm{N}_{2}$ concentrations during a single laser pulse. The precision of temperature determination critically depends on the reliability of the available data on line broadening coefficients and their dependences on temperature and concentrations of collision partners.

Hence the implementation of single-shot high-resolution lineshape CARS spectroscopy is still important. 


\section{EXPERIMENTAL}

In our previous experiments, ${ }^{4}$ we developed a highresolution spectroscopic technique that allows single-shot CARS spectra of hydrogen Q-branch molecular transitions to be obtained in non-stationary media with a resolution of $\sim 0.1 \mathrm{~cm}^{-1}$. The method is based on the application of a Fabry-Pérot interferometer to the spectral analysis of a broadband (BB) CARS spectral line profile with detection of an interference pattern by a linear charge-coupled device (CCD) detector. The error of the measured linewidth broadening coefficients was about $30 \%$.

The aim of the present work was to decrease the error of linewidth measurements. It was understood that this error was mainly due to the poor quality of recorded interference patterns. It has also been found that the main factor deteriorating the quality of interference patterns was the spectral noise of a dye laser.

In order to overcome this problem and increase the quality of single-shot spectral line contours, we applied a dual-broadband ${ }^{8,9}$ (DBB) CARS configuration. This approach reduces the spectral noise in the CARS lineshape obtained during a single laser shot by averaging over the spectrum of driving force (Raman pumps spectra convolution) resulting from the interaction of two broadband pump dye laser sources with Raman transition.

Speckle patterns existing in the plane of the CCD detector because of the multi-mode fiber used for transportation of CARS radiation also lead to additional noise in interferometer ring shapes. To reduce this part of the contribution to distortion of line profiles, detection of total Fabry-Pérot ring patterns with a two-dimensional CCD detector was applied and angular integration of CARS intensities during data processing was performed.

The scheme of the experimental set-up is shown in Fig. 1. To implement the DBB CARS approach, Raman excitation was performed by two 'relatively broad' dye lasers (pump and Stokes waves) having spectral linewidths of $\sim 10 \mathrm{~cm}^{-1}$. On the one hand these widths are small enough for their convolution to be less than the Q-branch line spacing $\left(20-100 \mathrm{~cm}^{-1}\right)$, and on the other hand large enough for efficient light interaction with Raman transition and spectral averaging over dye laser spectra. Single-frequency (linewidth $\sim 100 \mathrm{MHz}$ ) second harmonic radiation of a Coherent Radiation Infinity $\mathrm{Nd}^{3+}:$ YAG pulse laser was used as a second pump wave in the CARS process. Typically the output energy of the Infinity instrument at $532 \mathrm{~nm}$ in a $3 \mathrm{~ns}$ pulse was $100-200 \mathrm{~mJ}$. An energy of $10-15 \mathrm{~mJ}$ was used for the CARS pump and the rest of the energy was used for pumping of both dye lasers. One of the dye lasers was operated with pyridine ('red') in DMSO solution and had $3 \mathrm{~mJ}$ per pulse output energy. The second ('yellow') was operated with Rhodamine 6G in methanol solution with $2 \mathrm{~mJ}$ per pulse output energy. The 'red' laser was tunable so the difference between the central wavenumbers of the two dye lasers $\left(\sim 4000 \mathrm{~cm}^{-1}\right)$ could be tuned to Raman wavenumbers of individual $\mathrm{Q}_{1}-\mathrm{Q}_{9}$ lines of the hydrogen molecule.

All three laser beams were focused either in a highpressure pulsed burner or a cell containing the gas under static conditions. Single-shot DBB CARS spectra were generated at a repetition rate of $1 \mathrm{~Hz}$.

CARS spectra were analyzed with a Fabry-Pérot interferometer (see Fig. 1) having a free spectral range (FSR) of $1.67 \mathrm{~cm}^{-1}$ and finesse 12 . The interference rings were detected with a two-dimensional multichannel CCD detector. A specially developed software package read recorded interference patterns, calculated the coordinates of their center of symmetry and then performed angular averaging along the complete circumference of Fabry-Pérot rings.

\section{RESULTS AND DISCUSSION}

In our earlier experiments ${ }^{4}$ based on a broadband CARS configuration the second harmonic of a single-frequency $\mathrm{Nd}$ : YAG laser was used as a pump wave and dye laser light with a spectral width of $\sim 10 \mathrm{~cm}^{-1}$ was used as a Stokes wave. It was observed that independently of the dye laser design [we used both lasers with cavity mirrors (with different mode spacing) and 'cavityless' lasers], their spectral noise was significant and irreproducible from shot to shot. These noise irregularities were very broadband and had Fourier components of the same scale as the Raman linewidth itself and provided random spectral structures within individual CARS lineshapes which one can observe in a series of CARS interferograms, presented in Fig. 2. Moreover, many of the interference patterns were so noisy and modulated that it was impossible to use them in data processing routines. This forced us to exclude them from consideration, which in turn led to increased statistical errors in linewidth calculations.

As a result of our recent innovations that included a DBB CARS approach and two-dimensional detection of Fabry-Pérot rings with further angular integration of CARS intensities along the rings, nearly all of the single-shot

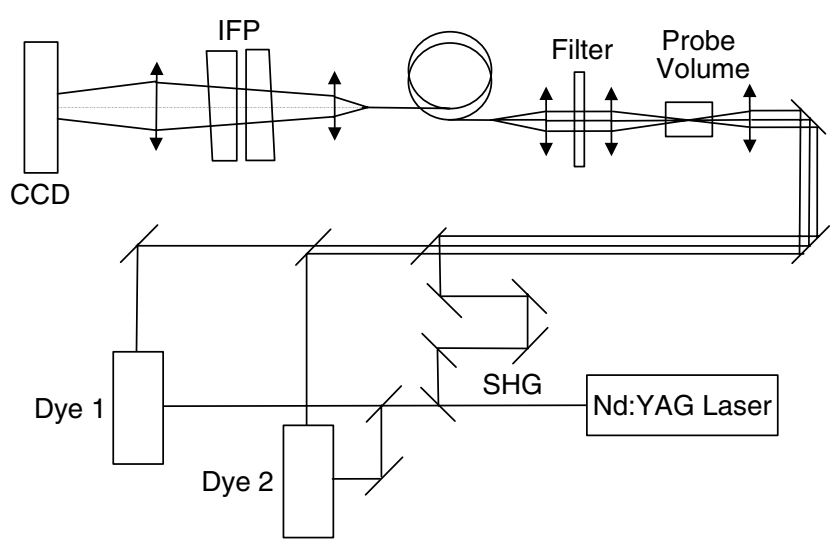

Figure 1. Optical layout of the experimental set-up. 

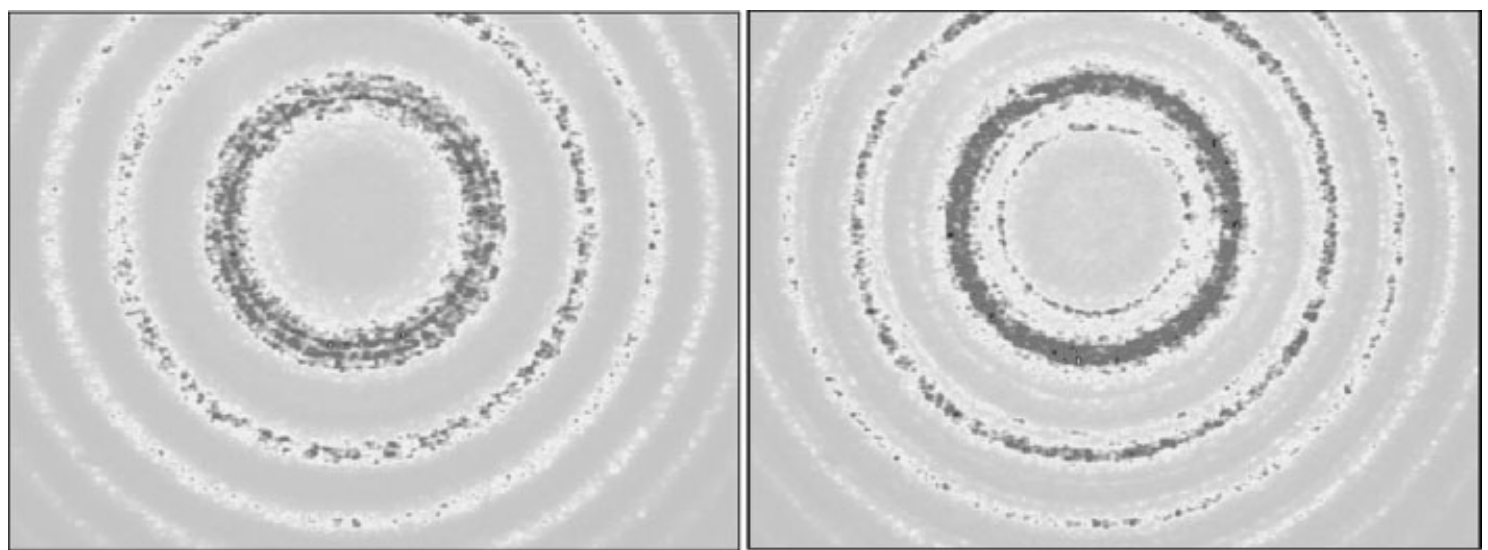

(a)

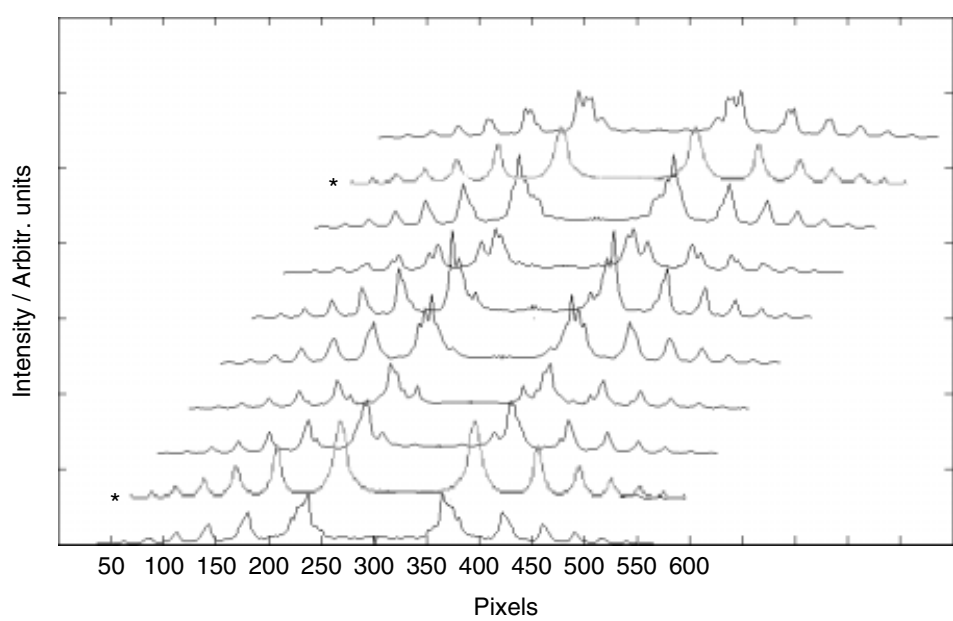

Figure 2. (a) Fabry-Pérot rings of two sequential BB CARS laser shots obtained in a cell with a gas mixture of $\mathrm{H}_{2}$ and $\mathrm{N}_{2}(3+42$ bar) at $300 \mathrm{~K}$. Chaotic two-dimensional ring speckle structure together with fluctuating substructure of interference rings which is due to shot-to-shot fluctuations of Stokes laser spectra are clearly seen. (b) Results of averaging along the circumference of Fabry-Pérot rings for a series of 10 laser shots obtained in a BB CARS configuration in a cell with a gas mixture of $\mathrm{H}_{2}$ and $\mathrm{N}_{2}(3+42$ bar) at $300 \mathrm{~K}$. Chaotic modulation of spectral lines due to shot-to-shot fluctuations of Stokes laser spectra is clearly seen. Significant (marked with asterisks) spectra selection is necessary.

interference patterns obtained in our experiments became valid for linewidth measurements (Fig. 3).

In order to compare directly these two approaches (for both $\mathrm{BB}$ and $\mathrm{DBB}$ configurations), $\mathrm{Q}_{1}$ linewidth measurements were performed under the same conditions in a cell containing a mixture of $\mathrm{H}_{2}$ and $\mathrm{N}_{2}$ at 1 and 40 bar respectively. In both cases the width of the slit function was $0.09 \mathrm{~cm}^{-1}$. Figure 4 presents histograms of $\mathrm{H}_{2} \mathrm{Q}_{1}$ linewidth measurements for the $\mathrm{BB}$ and $\mathrm{DBB}$ configurations. The average linewidth is $0.16 \mathrm{~cm}^{-1}$; the histogram width for DBB CARS is $0.006 \mathrm{~cm}^{-1}$ and for BB CARS $0.017 \mathrm{~cm}^{-1}$. Hence application of a DBB CARS configuration allows much better precision of linewidth measurements.

Based on the above results, the following approach can be suggested for single laser shot temperature measurements in flames with hydrocarbon fuel where hydrogen is used as a probe molecule. The idea involves the simultaneous detection of hydrogen Q-branch spectra with the help of a spectrometer (for temperature measurement) and an interferometer (for linewidth measurement) having orthogonal dispersions. A similar optical schematic was successfully implemented for $\mathrm{D}_{2}$ density measurements in a cell in a mixture with argon. ${ }^{10}$ In such an approach, the information on linewidths obtained from a singleshot DBB CARS spectrum is directly used in temperature evaluation routines and does not require knowledge of species concentration and medium density.

\section{CONCLUSIONS}

The implementation of the DBB CARS configuration with spectral averaging over pump and Stokes laser spectra together with angular averaging of CARS intensities along the complete circumference of Fabry-Pérot interference patterns leads to a significant improvement of interferogram 

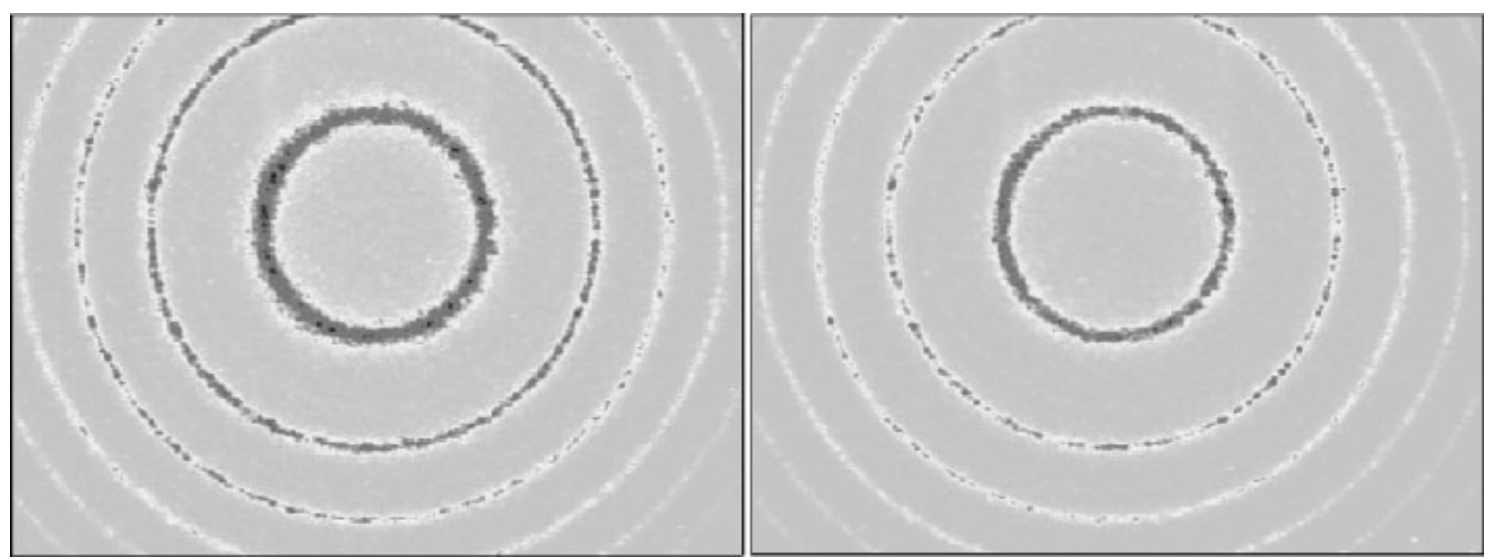

(a)

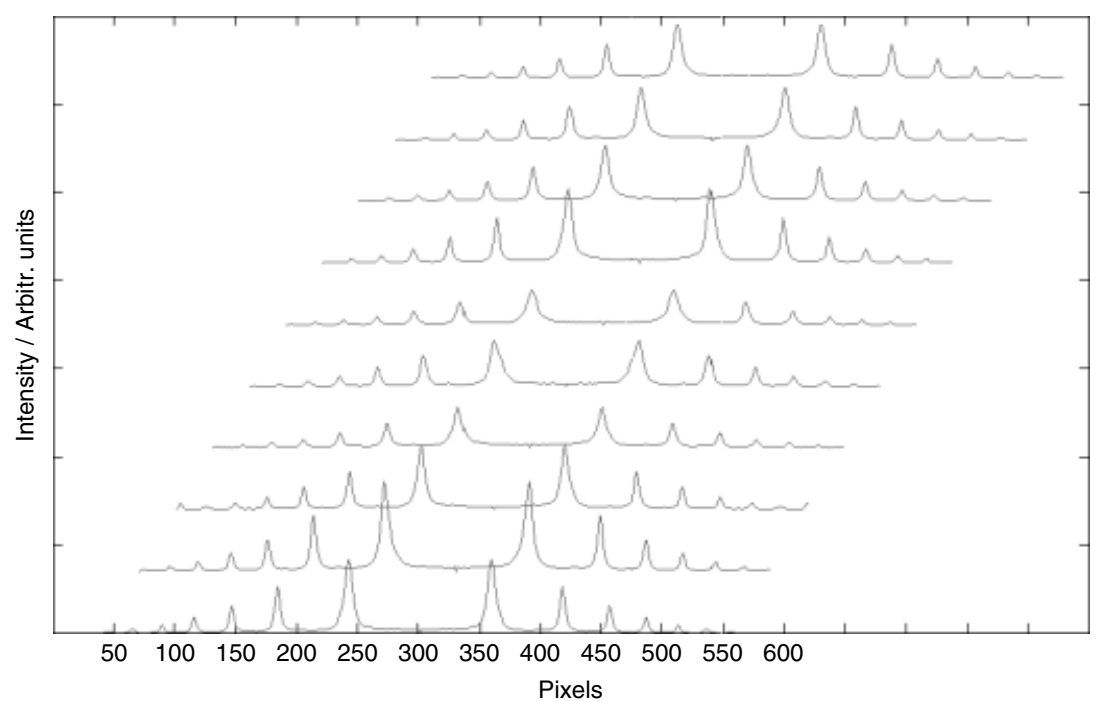

(b)

Figure 3. (a) Fabry-Pérot rings of two sequential DBB CARS laser shots, obtained in a cell with a gas mixture of $\mathrm{H}_{2}$ and $\mathrm{N}_{2}(3+14$ bar) at $300 \mathrm{~K}$. Only a chaotic speckle structure is seen. (b) Results of averaging along the circumference of Fabry-Pérot rings for a series of 10 laser shots, obtained in a DBB CARS configuration in a cell with a gas mixture of $\mathrm{H}_{2}$ and $\mathrm{N}_{2}(3+14$ bar) at $300 \mathrm{~K}$. All spectral lines have good quality and are suitable for further evaluation.
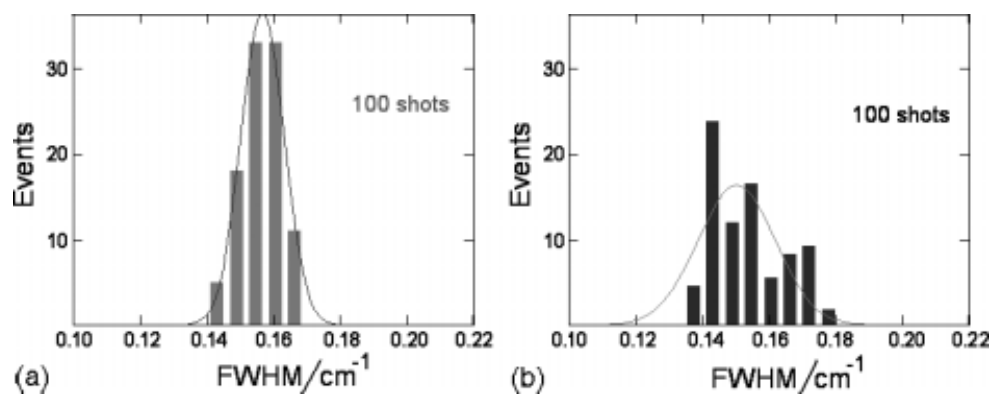

Figure 4. Histograms of $\mathrm{H}_{2} \mathrm{Q}_{1}$ linewidth measurements for (a) DBB and (b) BB configurations under the same experimental conditions. Gas mixture of $\mathrm{H}_{2}$ and $\mathrm{N}_{2}\left(1+40\right.$ bar) at $300 \mathrm{~K}$. Average linewidth is $0.16 \mathrm{~cm}^{-1}$; histogram width for DBB CARS is $0.006 \mathrm{~cm}^{-1}$ and for BB CARS $0.017 \mathrm{~cm}^{-1}$.

quality obtained for single laser shot CARS hydrogen Qbranch linewidth measurements. Nearly all of the singleshot DBB CARS signal interference patterns obtained in a pulsed high-pressure burner became suitable for linewidth determination. The results obtained also allow the conclusion to be drawn that this approach is suitable not only for building up schematics of $\mathrm{H}_{2} / \mathrm{O}_{2}$ CARS thermometry, but also for flame investigations where hydrocarbons are used as a fuel and air or oxygen as an oxidizer. 


\section{REFERENCES}

1. Bergmann V, Stricker W. Appl. Phys. B 1995; 61: 49.

2. Hussong J, Lueckerath $R$, Stricker $W$, Bruet $X$, Joubert $P$, Bonamy J, Robert D. Appl. Phys. B 2001; 73: 165.

3. Gristi F, Bouchardy P, Vingert L, Clauss W, Oschwald M, Smirnov V. CARS measurements in Cryogenic LOX-GH2 Jet Flames, Liquid Rocket Thrust Chambers: Aspects of Modelling Analysis and Design. In Progress in Astronautics and Aeronautics, Yang V, Habiballah M, Hulka J, Popp M (eds). 2005; Chapt. 10.

4. Clauss W, Klimenko DN, Oschwald M, Vereschagin KA, Smirnov VV, Stelmakh OM, Fabelinsky VI. J. Raman Spectrosc. 2002; 33: 906.
5. Berger J-P. Thèse de Doctorat, Université de Bourgogne, Dijon, 1994.

6. Michaut X, Berger J-P, Saint-Loup R, Chaussard F, Berger H. In Combustion dans les Moteurs Fusées, 26-28 June 2001, Actes $d u$ Collogue de Synthese $d u$ Groupe de Recherche CNES/CNRS/ONERA/SNEGMA. Cepadues Editions: Toulouse, $2001 ; 321$.

7. Shaussard F, Michaut X, Saint-Loup R, Berger H, Joubert $P$, Lance B, Bonamy J, Robert D. J. Chem. Phys. 2000; 112: 158.

8. Alden M, Bengtsson P-E, Edner H. Appl. Opt. 1986; 25: 4493.

9. Eckbreth AC, Anderson TJ. Opt. Lett. 1986; 11: 496.

10. Gillespie WD, Hahn JW, Bowers WJ, Hurst WS Jr, Rosasco GJ. Appl. Opt. 1999; 38: 534. 